\title{
What is the Maximun IR Luminosity of a Single Spiral Galaxy?
}

\author{
A. M. Mickaelian \\ Byurakan Astrophysical Observatory and Isaac Newton Institute \\ Armenian Branch, 378433 Byurakan, Armenia \\ M.-P. Véron-Cetty and P. Véron \\ Observatoire de Haute Provence, CNRS, F-04870 Saint-Michel \\ l'Observatoire, France
}

\begin{abstract}
.
A new sample of IRAS galaxies at high galactic latitudes has been built by optically identifying IRAS point sources.

In no more than about 100 cases, an apparently interacting system of galaxies is located near the IR source. The large majority are unambiguously identified with a single, relatively isolated galaxy. This confirms that, although galaxy interaction may trigger starburst activity, the IR luminosity in most objects is due to normal star formation in the disc.
\end{abstract}

\section{Introduction}

Most IRAS galaxies are relatively normal, isolated spirals; their IR luminosity is consistent with normal rates of star formation in their disc (Moorwood, VéronCetty, \& Glass 1987). However, at luminosity above $10^{11} \mathrm{~L}_{\odot}$, the trigger for the intense IR emission is the strong interaction/merger of molecular gas-rich spi rals (Sanders 1997). We have carried out a program of optical identification for a large sample of IRAS point sources in order to evaluate which IR luminosity a single spiral galaxy may reach.

\section{The sample}

A program of optical identification of all sources in the IRAS Point Source Cata$\log$ (Beichman et al. 1988) in a $1530 \mathrm{deg}^{2}$ area $\left(\delta>61^{\circ}\right)$ at high galactic latitude $\left(|b|>15^{\circ}\right)$ was initiated in 1995 at Byurakan Observatory (Mickaelian 1995). The sample contains 3788 IRAS sources; 1821 have starlike colors $\left(\mathrm{S}_{60} / \mathrm{S}_{12}<3\right.$, Meiskin \& Davis 1986); 454 others are flagged in the IRAS catalogue as possible cirrus; they have been ignored. We are left with a sample of 1513 probable extragalactic objects (constituting the Byurakan-IRAS Galaxy (BIG) sample), of which 873 have published identifications. We searched for an optical counterpart on the FBS plates and on the Digital Sky Survey images (DSS) for the 640 others (Mickaelian 2000, and references therein). 
Cross-correlation between the IRAS PSC and NVSS (Condon et al. 1998) catalogs yielded 853 coincidences within $30^{\prime \prime}$ (from the sample of 1513 IRAS sources), of which only about 20 are chance coincidences; the better accuracy of the NVSS positions, facilitated the identification process.

A rough classification has been made for each optical candidate. Some 150 sources have no optical counterpart or are associated with a very weak object; they are probably optically very faint galaxies. Most of the galaxies are isolated galaxies ( $\mathrm{S} 0$ or later), although a small number of sources have been identified with what seems to be an interacting system of galaxies.

\section{Conclusions}

We estimate that no more than about 100 IRAS sources are associated with interacting or merging systems. The large majority are unambiguously identified with a single, relatively isolated galaxy. This confirms the earlier finding that the IR luminosity of most IRAS galaxies is due to normal star formation activity in their disc.

A program has been undertaken to measure the redshift of a subsample of these objects to determine what is the maximum IR luminosity of an isolated galaxy; it must be smaller than $10^{11} \mathrm{~L}_{\odot}$ as, above this value, all IR sources seem to be interacting systems (Sanders 1997).

Acknowledgments. The authors have used the NASA/IPAC Extragalactic Database (NED) operated by the Jet Propulsion Laboratory (Caltech), under contract with the National Aeronautics and Space Administration. The Digitized Sky Survey was produced at the Space Telescope Science Institute (STScI) under U.S. Government grant NAG W-2166.

\section{References}

Beichman, C.A., Neugebauer, G., Habing, H.J., Clegg, P.E., Chester, T.J. 1988, Infrared Astronomical Satellite (IRAS), Catalogs and atlases, The Point Source Catalog, NASA. Washington D.C.

Condon, J.J., Cotton, W.D., Greisen, E.W., Yin, Q.F., Perley, R.A., Taylor, G.B., Broderick, J.J. 1998, AJ, 115, 1693

Meiskin, A., Davis, M. 1986, AJ, 91, 191

Mickaelian, A.M. 1995, Afz, 38, 625

Mickaelian, A.M. 2000, Afz, 43, 425

Moorwood, A.F.M., Véron-Cetty, M.-P., Glass, I.S. 1987, A\&A, 184, 63

Sanders, D.B. 1997, Rev. Mex AA (Serie de Conferencias), 6, 42 